\title{
BAD ROAD DETECTION AND WARNING SYSTEM USING LASER SENSOR
}

\author{
Etukala Neeraganti Sai Kaushik ${ }^{1}$, Guruprasad Hebbar ${ }^{2}$, Deekshith M T $^{3}$, Kumar Mani Chandra $\mathbf{Y}^{4}$ \\ ${ }^{I}$ Student, Dept. of Electronics \& Communication, BNM Institute of Technology, Karnataka, India \\ ${ }^{2}$ Student, Dept. of Electronics \& Communication, BNM Institute of Technology, Karnataka, India \\ ${ }^{3}$ Student, Dept. of Electronics \& Communication, BNM Institute of Technology, Karnataka, India \\ ${ }^{4}$ Student, Dept. of Electronics \& Communication, BNM Institute of Technology, Karnataka, India
}

\begin{abstract}
Laser can travel large distances without getting deviated and it exhibits the property of reflection. Based on these properties measuring distance using time of flight concept is possible. By determining the distances we can implement a system which can detects the bad roads like potholes, damaged roads and speed breakers and give warning to the driver. In this paper we have also discussed on how effectively this system works in real world, challenges and how it overcomes them.
\end{abstract}

Keywords: Laser; Time of flight; Distance measurement; Bad road detection; Potholes detection; Speed breaker detection.

\section{INTRODUCTION}

India has the world's third largest road network with 5 million kilometers on of 2016 but the underlying fact is that most of these roads are in poor condition. Out of 5 million kilometers 960,000 kilometers are surfaced roads and about 1 million kilometers of road in India are the poorly constructed ones [1].

The condition of the roads is one of the main reasons for the accidents which are occurring. According to a survey by Times of India, over 10,000 people lost their lives by road accidents due to bad roads in 2015 in India. Bad roads can be of anything like damaged roads, speed breakers and potholes. Out of those 10,000 people who have died by bad roads, 3416 people have died due to the potholes. So we need an efficient system which can detect the bad roads and immediately give a warning to the driver[1].

In this system we are going to detect the bad roads in terms of change in distance. The device is placed over the automobile in a certain angle so that it is directed towards the road as shown in the fig 1

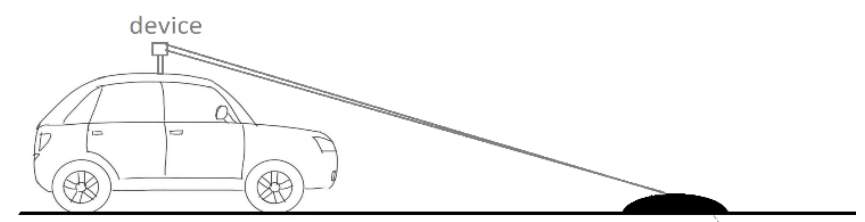

speed breaker

Fig - 1: Position of the device on car

The device continuously measures the distance between the device and road, a sudden change in the distance determines the bad road. In case of speed breaker the distance first decreases and comes to constant value and in case of pothole the distance first decreases and then comes to normal value, all this change in distances will happen in fraction of seconds since automobile will be travelling with certain speed. Any these kind of change in distances indicates the bad roads so an immediate warning will be given in the form of sound to the driver. All the real world scenarios like elevated roads and depressed roads, and differentiating between an automobile before the device and bad roads are discussed further in this paper. We are using DML 40-2-1111 [4] distance measuring sensor based on laser, Arduinouno for microcontroller, a piezo buzzer for alarm.

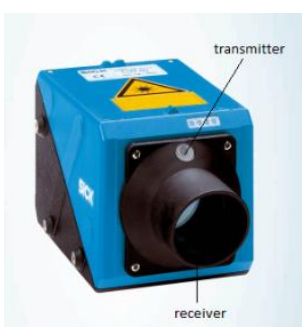

Fig -2: DML 40-2-1111

\section{DML 40-2 1111}

The device shown in the fig 1 [4] is used for measuring distance. It consists of transmitter and receiver. It sends a laser light using the transmitter, the light wave travels and hits the obstacle and gets reflected back. The reflected wave is sensed by the receiver. The total time taken by the laser to travel from the transmitter to obstacle and from the obstacle to the receiver is defined as Time of flight. The time of flight multiplied by the speed of light in air gives the distance. 


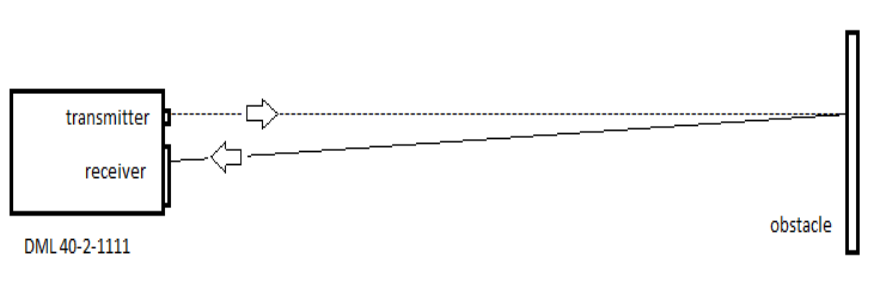

Fig-3: Principle of distance measuring

The measurement cycle time for the sensor is $0.195 \mathrm{~ms}$ and the response time is $1 \mathrm{~ms}$. So the distance is measured is continuously and the chance of not detecting the bad road is very low. The angle between the incident ray and the reflected ray is almost zero and the both rays almost co inside with each other, Hence there is no need of considering the angle between and the measured distance is given by

Speed of light $=\mathrm{c} /$ refractive index of the medium

Speed of light in air $=$ c/refractive index of air

Speed of light in air $=\left(2.99 * 10^{\wedge} 8\right) / 1.003=2.981 * 10^{\wedge} 8 \mathrm{~m} / \mathrm{s}$

Measured distance is given as

$$
\mathrm{m}=\left(\left(2.981 * 10^{\wedge} 8\right) *(\text { time of flight })\right) / 2
$$

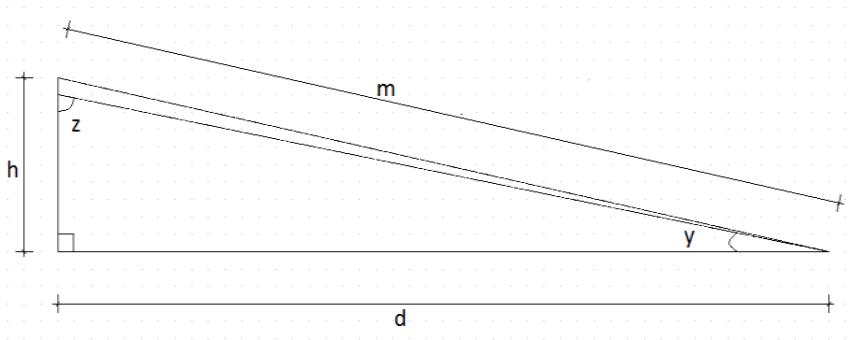

Fig-4: Representing the action rage

Here measured distance " $\mathrm{m}$ " is not the actual range before which the system give alarm. The measured distance and range is differentiated in the Fig.4. The range is given by "d".

"h" denotes the height at which system is placed (on the hood of the car), " $y$ " denotes the angle between road and reflected ray and "z" gives the angle at which the system should be placed for the corresponding desired range. In general, the average height of the car is around $1750 \mathrm{~mm}$ and this device is kept over the roof of the car resulting an average height of $2000 \mathrm{~mm}$ or 2 meters. Let us say the device is designed to detect the bad road which is 100 meters away, then the measured distance is given by

$$
\mathrm{m}=\operatorname{sqrt}\left(\mathrm{d}^{\wedge} 2+\mathrm{h}^{\wedge} 2\right)
$$

$\mathrm{h}=2 \mathrm{~m}, \mathrm{~d}=100 \mathrm{~m}$

We get $\mathrm{m}=100.01 \mathrm{~m}$

So, when we consider the height the value we get is 100.01 for the actual distance of 100 . The error occurred due to the height is $0.01 \mathrm{~m}$ for $100 \mathrm{~m}$. Which is $0.01 \%$. Since the error is very small the height of the car and the height at which the device is placed can be neglected. From now we can assume

$$
\mathrm{m}=\mathrm{d}
$$

During the time of flight of laser, car also would have moved certain distance, since we have set the range for 100 meters, we have to calculate the distance moved by the car during the time of flight.

Time taken for the laser to travel 100 meters

$$
\mathrm{T}_{1}=100 /\left(2.981 * 10^{\wedge} 8\right)
$$

$\mathrm{T}_{1}=0.335$ micro seconds

In 0.335 micro seconds car would have moved very less distance, for a car which is moving at $100 \mathrm{kmph}$, the distance covered in 0.335 micro second is of 0.0335 meters, which is very less. So the distance covered by the car in the meantime can be neglected.

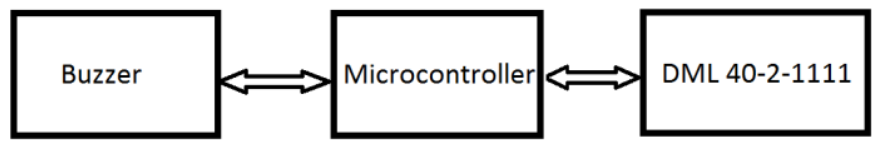

Fig-5: Block diagram

DML 40-2-1111 is connected to Microcontroller, when the transmitter sends the signal then microcontroller starts counter and when the receiver receives the reflected signal then microcontroller stops the signal. The counted time is called the time of flight and range is calculated using the above mentioned formulas. The range remains constant during good roads, when a speed breaker or the pothole is detected then the distance varies in fraction of seconds. Then microcontroller sends the signal to buzzer. DML 40-21111 supports RS 232 serial communication and it has serial communication port. The connections are given as shown in Fig.6.

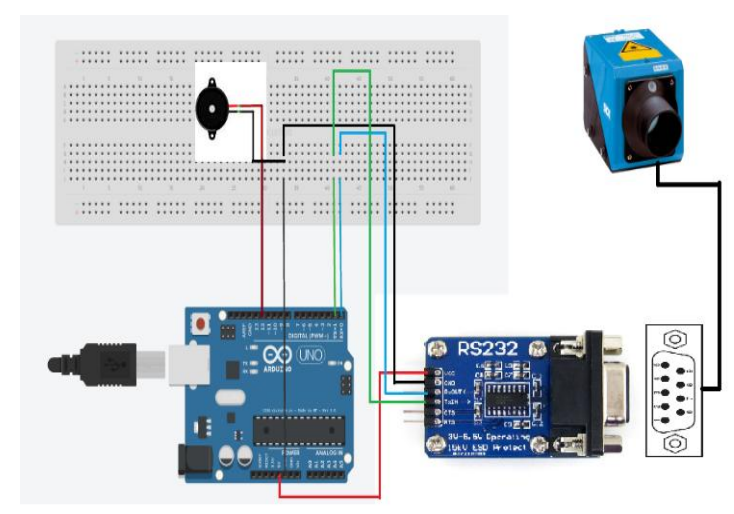


Pin 12 of micro controller to buzzer

Pin 0 of microcontroller to RX (pin 2) of RS232

Pin 1 of microcontroller to TX (pin 3) of RS232

GND of microcontroller to GND (pin 5) of RS232

$5 \mathrm{v}$ pin of microcontroller to pin 9 of RS232. The other side of the RS232 to the DML 40-2-1111. Otherwise we can simply use an RS232 to usb converter and keeping the micro controller in serial mode we can directly connect DML 402-1111 to microcontroller.

The challenges for this system are when there is another vehicle in front of it and when the road is either in steep up or down. When there is a vehicle in front of the system the distance changes since the vehicle obstructs the ray from hitting the road, then the measured distance by the device is changed and gives alarm which is not desired, so the programming should be done such a way that an vehicle should not be considered as bad road. If there a vehicle in front of the system, then the distance changes suddenly changes (drops) and it will remain in that range as long as the vehicle is in front of it but in the case of bad road the distance suddenly changes and comes to normal, by this way a vehicle is differentiated from the bad road. The following graph demonstrate the response when a vehicle is detected and when a vehicle is detected.

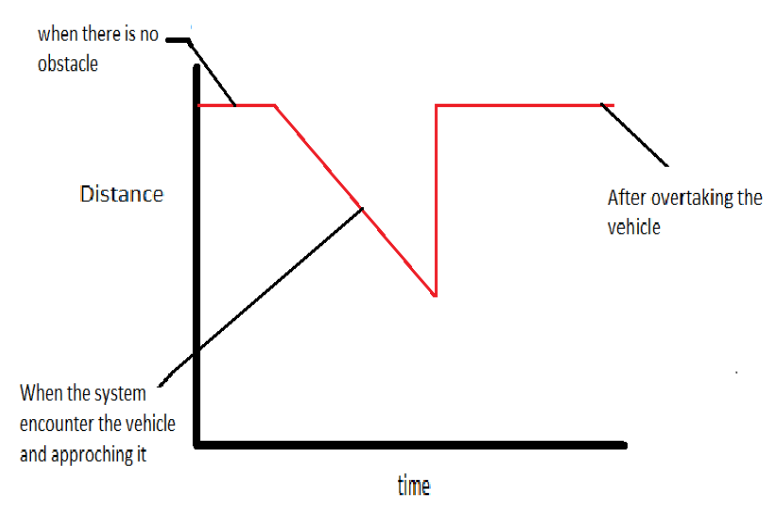

Graph-1: Response when vehicle is detected

Second challenge is when the road is steep, it can be inclining or declining. In the case of inclining road, the change in distance is gradually decreasing and in case of declining roads the distance will be gradually decreased and the system should not detect these as the bad road. The following graph shows the response when the roads are inclining or declining.
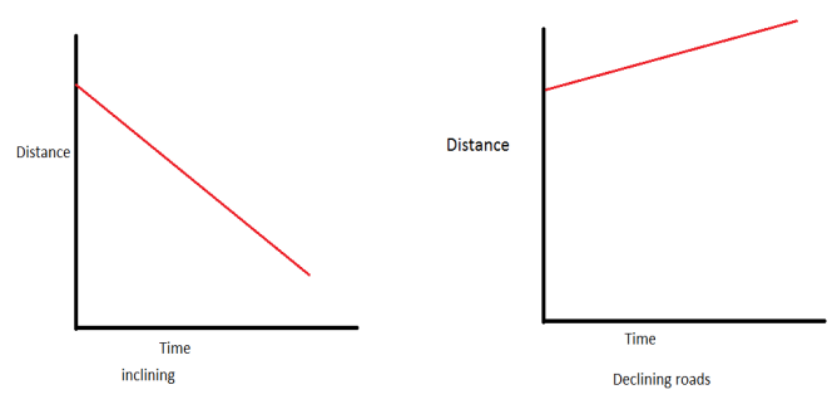

Graph-2: Inclining and declining roads

The following graph shows the response when the system encounters the bad road

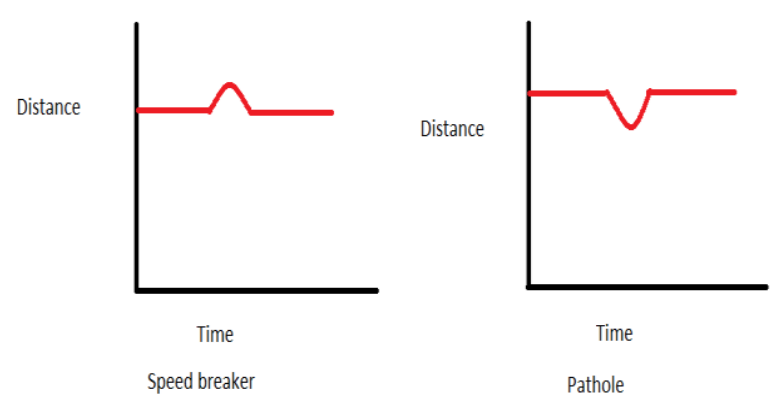

Graph-3: Speed breaker and pothole detection

To overcome these problems we have designed an algorithm which can distinguishes between bad road, a vehicle and a inclining or declining road. The distance for which we are designing is $100 \mathrm{~m}$. The measured values will be comparing with the fixed value $100 \mathrm{~m}$. If the deviation is occurred in the small amount of time then it is considered as bad road. If the distance is constantly decreasing then it is considered as inclining road. If the distance keeps constantly increasing then the road is considered as decreasing. In the case of encountering a vehicle in front of the system the distance keeps reducing and then suddenly increases. Since it is as same as inclining road. This case will rule out.

The algorithm for detecting the bad road and differentiating it from vehicles and inclining and declining roads is as shown below. Here we are designing the range for 100 meters, before which it gives alarm. It constantly measures the distance and compared to 100. If there are any deflections, then it will wait for 1 second and measures again. If we get the distance as 100 now, then it is considered as bad road otherwise it can be any of other three cases as discussed and explained through graphs. 


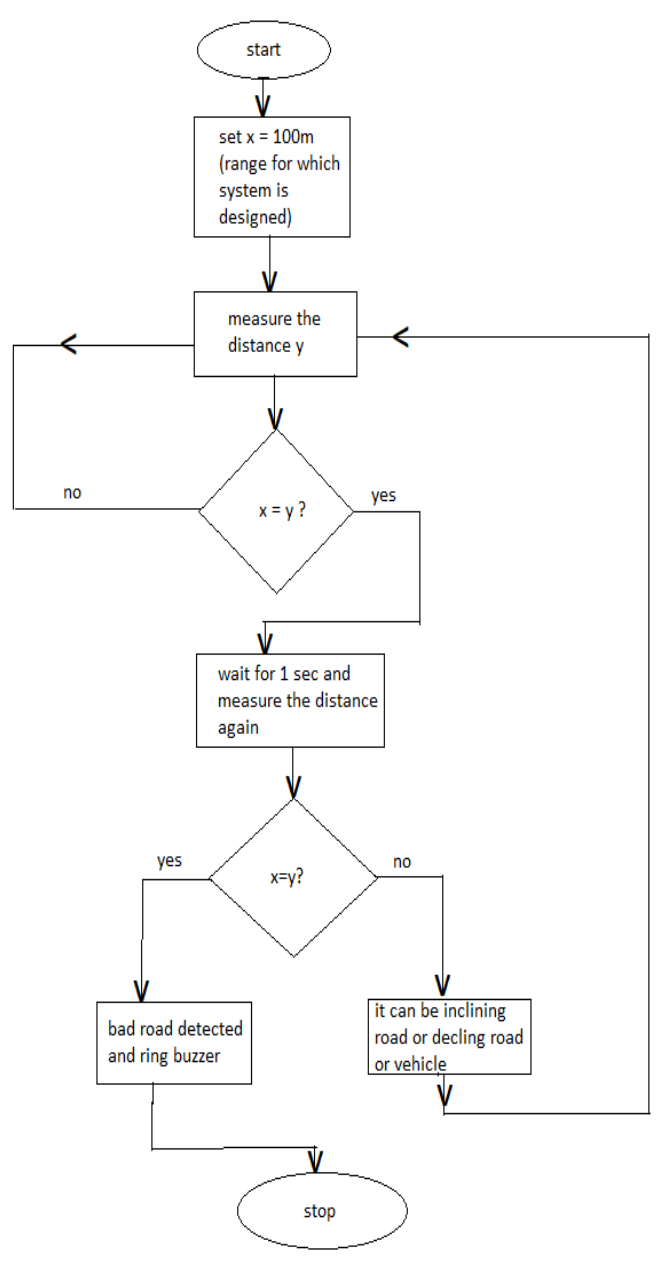

Fig-7: flow chart

\section{FUTURE SCOPE}

This system will be very useful particularly in the countries like india. In future It can be integrated with gps so that the gps co-ordinates will be uploaded to the google maps such that it when a vehicle without this system but using the google maps also gets the warning when they are near to it and also it will be very helpful for the R\&B department to identify and repair the road. Once they are repaired tag will be removed by them.

\section{CONCLUSION}

The system is simple and can be installed in any kind of car. By the system we can save many lives that are being lost due to the accidents occurring due to bad roads by giving warning before 100 meters itself. By integrating the model with the GPS and android apps it can be used even more efficient way.

\section{REFERENCES}

[1]. http://timesofindia.indiatimes.com/india/Bad-roadskilled-over-10k-people-in-2015-3416-deaths-due-topotholes/articleshow/53482615.cms
[2]. C C Wang, C Thorpe and A Suppe, 'Ladar-Based Detection and Tracking of Moving Objects from a Ground Vehicle at High Speeds', IEEE Intelligent Vehicles Symposium (IV2003), 2003.

[3]. H Cramer and G Wanielik, 'Road border detection and tracking in noncooperative areas with a laser radar system', Proc. German Radar Symp., Bonn, Germany, 2002 24-29. [4]. www.sick.com/in/en

\section{BIOGRAPHIES}

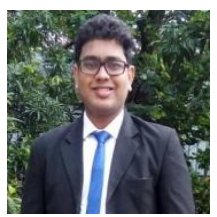

ETUKALA NEERUGANTI SAI KAUSHIK hails from Bangalore, India. He is pursuing $\mathrm{BE}$ in Electronics and Communication Engineering. His Research interests include Embedded Systems and VLSI.

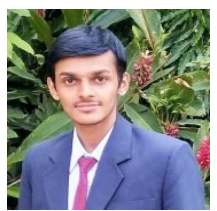

GURUPRASAD HEBBAR hails from Bangalore, India. $\mathrm{He}$ is pursuing $\mathrm{BE}$ in Electronics and Communication Engineering. His Research interests include Embedded Systems and VLSI.

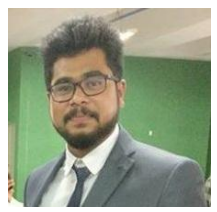

DEEKSHITH M T hails from Bangalore, India. He is pursuing BE in Electronics and Communication Engineering. His Research interests include Embedded Systems and Networks.

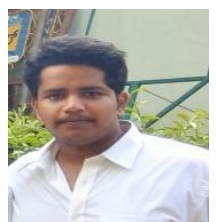

KUMAR MANI CHANDRA Y hails from Bangalore, India. $\mathrm{He}$ is pursuing $\mathrm{BE}$ in Electronics and Communication Engineering. His Research interests include Image Processing and Signal Processing. 